\title{
Diferencias en los estilos de aprendizaje desde la escuela primaria hasta el posgrado en medicina
}

\author{
Raúl A. Borracci, Diego Manente, Susana Tamini, Mario Dvorkin, Eduardo B. Arribalzaga, Hugo Grancelli, \\ Amanda Galli
}

Objetivos. Describir los estilos de aprendizaje de estudiantes de medicina al ingreso y en el posgrado inmediato, de acuerdo con las modalidades preferidas para usar la información, y comparar estos resultados con los estilos de alumnos de la escuela primaria, a fin de hallar diferencias generacionales.

Sujetos y métodos. Se administró el cuestionario VARK a 73 alumnos de séptimo grado de una escuela primaria, 113 estudiantes de primer año de medicina y 141 residentes. Se realizó un análisis exploratorio para determinar conjuntos con características afines (análisis de conglomerados). El conteo y suma de los resultados de cada individuo representó el estilo preponderante de cada grupo, definiéndose como dominante cuando se elegía en más del 50\% de las respuestas.

Resultados. Los estilos preponderantes en los tres grupos fueron cinestésico $(27,5 \%)$ y bimodal $(37,3 \%)$, mientras que el lectoescritor creció desde la escuela primaria hasta el posgrado (1,4\% a 10,7\%; $p=0.037)$, con una tendencia de la caída del estilo auditivo desde la primaria hasta la universidad y posgrado (19,2\% a 12,2\%; $p=0.269)$. Para el estilo bimodal, la combinación preponderante fue auditivo-cinestésica, que disminuyó desde la primaria hasta el posgrado $(65 \%$ a $31 \% ; p=0,002)$.

Conclusiones. El análisis de correspondencia mostró que los alumnos de la escuela primaria preferían el estilo auditivo, los universitarios se acercaban al cinestésico y los de posgrado tenían una preferencia intermedia entre el visual y el lectoescritor. Este último perfil creció significativamente desde la escuela primaria hasta el posgrado, con una tendencia inversa del estilo auditivo y del bimodal auditivo-cinestésico entre los mismos grupos.

Palabras clave. Cuestionarios. Educación médica. Estilos de aprendizaje. Estudiantes de medicina.

Departamento de Bioestadística; Facultad de Ciencias Biomédicas; Universidad Austral (R.A. Borracci, E.B. Arribalzaga). Área de Investigación; Sociedad Argentina de Cardiología (D. Manente, S. Tamini, H. Grancelli, A. Galli). Departamento de Fisiología; Facultad de Medicina; Universidad de Buenos Aires (M. Dvorkin). Buenos Aires, Argentina.

Correspondencia:

Dr. Raúl A. Borracci.

La Pampa, 3030. 1428 Buenos Aires (Argentina).

E-mail:

raborracci@gmail.com

Conflicto de intereses: No declarado.

Competing interests: None declared.

(c) 2015 FEM

\section{Learning styles preferences from elementary school to medical postgraduate}

Aims. To describe learning styles observed in first-year medical students and in postgraduates, regarding sensory preferences to use information, and to compare the results with the learning styles of last-year elementary school pupils, in order to find out generational differences.

Subjects and methods. The VARK questionnaire was administered to 73 last-year elementary school pupils, 113 first-year medical students and 141 residents of cardiology (postgraduate). The questionnaire consists of 16 questions and each answer was associated to a particular learning style, corresponding to visual, aural, reading/writing or kinaesthetic.

Results. Most common styles were kinaesthetic (27.5\%) and bimodal (37.3\%) in the three groups, with some differences in reading/writing profile that increased from elementary school up to postgraduate (1.4\% to $10.7 \% ; p=0.037$ ), and a slight tendency to decrease aural style from that educational level to the university and the postgraduate $(19.2 \%$ to $12.2 \% ; p=$ 0.269). Within bimodal styles, the most common combination was aural-kinaesthetic, that decreased from the elementary school to the postgraduate ( $65 \%$ to $31 \% ; p=0.002)$.

Conclusions. Correspondence analysis showed last-year elementary school pupils preferred aural style, medical students were nearer to kinaesthetic mode, and postgraduates had an intermediate preference between visual and reading/writing styles. The last profile significantly increased from elementary school to postgraduate, with an inverse tendency of aural and aural-kinaesthetic bimodal styles among the two same levels.

Key words. Medical education. Medical students. Questionnaire. Research methods. 


\section{Introducción}

La motivación y el desempeño de los estudiantes suele relacionarse, entre otras cosas, con los estilos de aprendizaje, así como también con el empleo que hagan los docentes de estas preferencias [1]. Las investigaciones en el área de la teoría del aprendizaje han demostrado que las personas aprenden de diversas formas, y una de las variables más importantes que influyen en esta capacidad es justamente el estilo de aprendizaje, que indica la tendencia a desarrollar o elegir ciertas estrategias de aprendizaje sobre otras [2]. Por su parte, estas estrategias dependen fundamentalmente de la manera de adquirir, transmitir y usar la información.

La investigación de los estilos de aprendizaje y los tipos psicológicos es actualmente objeto de estudio en numerosas escuelas de medicina, dada su presunta relación con el desempeño académico, la elección de la especialidad y la satisfacción y eficiencia de los alumnos [3-12]. Además, el hecho de conocer el estilo de aprendizaje preponderante en un grupo podría servir para optimizar y potenciar los recursos que mejor incentiven el proceso de enseñanza.

Una forma de caracterizar los estilos de aprendizaje es determinar las modalidades sensoriales preferidas por los educandos para adquirir y transmitir la información nueva. Las cuatro formas sensoriales mayores son la visual, la auditiva, la cinestésica y la lectoescritora. Aunque los estudiantes pueden usar todas estas modalidades en conjunto, habitualmente existen uno o más modos preferentes o dominantes. Los individuos con perfil visual se inclinan más por el uso de diagramas, gráficos y símbolos, y es éste el estilo preponderante en los estudiantes de arte, diseño o informática. Los aprendices auditivos aprenden mejor escuchando clases y explorando y discutiendo las ideas. Por su parte, los estudiantes de humanidades suelen preferir el perfil lectoescritor, en el que interactúan más con textos, mientras que, por el contrario, los estudiantes de enfermería se inclinan por lo cinestésico, que incluye la experiencia manual directa o el denominado 'aprender haciendo'. Finalmente, los estudiantes de ciencia suelen presentar un perfil multimodal $[6,13]$. El cuestionario VARK (Visual, Aural, Read/Write, Kinesthetic), desarrollado por Fleming [13], es una herramienta que permite determinar las preferencias o el perfil de aprendizaje de los encuestados, en particular, la forma en que éstos adquieren o entregan la información desde su modalidad sensorial.

La mayoría de las investigaciones sobre estilos de aprendizaje en el nivel universitario destacan el per- fil promedio del grupo estudiado y la posibilidad de influir sobre él, en vista a modificar la preponderancia de algún estilo en especial $[6,7,14,15]$. Otros aspectos importantes lo constituyen las diferencias de estilo que pudieran darse entre estudiantes universitarios de los primeros años y aquellos a punto de graduarse o recientemente graduados $[16,17]$. En el caso del desempeño durante la carrera, podrían existir resultados antagónicos si el estilo de aprendizaje fuera preponderantemente cinestésico, y el de enseñanza o evaluación tuviera un perfil visual, o viceversa. De la misma forma, el aprendizaje de cada materia en particular podría requerir un estilo en especial.

Dado que conocer las preferencias para adquirir información podría ser útil para desarrollar estrategias acordes a los perfiles o estilos de los educandos, y habida cuenta de que existe poca información local en el ámbito de la carrera de medicina sobre las modalidades sensoriales de aprendizaje preferencial de los alumnos, se desarrolló el siguiente estudio.

El objetivo de este trabajo fue describir los distintos estilos de aprendizaje a los que recurren los estudiantes de medicina al ingreso en la universidad y en el posgrado, en particular, las modalidades sensoriales preferidas para adquirir y transmitir la información. Adicionalmente se compararon estos resultados con los estilos preponderantes hallados en alumnos que terminaban la escuela primaria, a fin de hallar diferencias generacionales o relacionadas con la edad.

\section{Sujetos y métodos}

Entre octubre y noviembre de 2010 se administró la versión española del cuestionario VARK a 73 alumnos de séptimo grado de una escuela primaria de la ciudad de Buenos Aires, a 113 estudiantes de primer año de la carrera de medicina durante su curso de fisiología y a 141 residentes de cardiología. Los encuestados respondieron de forma anónima y voluntaria el cuestionario, que se entregó en formato impreso a los distintos grupos. La tasa de respuesta completa del cuestionario fue del $93 \%$ en la escuela primaria, $80 \%$ en los estudiantes de medicina y $87 \%$ en los graduados. La alta tasa de respuesta se debió a que se eligió el momento del curso para completar el cuestionario, tanto en la escuela primaria como en la universidad. En el caso de los residentes, la encuesta se efectuó durante la concurrencia al curso superior de cardiología realizado en la sede de la Sociedad Argentina de Cardiología. El cuestionario, 
que puede obtenerse libremente, consta de 16 preguntas con cuatro opciones no excluyentes cada una. Cada respuesta se asocia a un estilo de aprendizaje que lo caracteriza: V (visual), A (auditivo), R (lectoescritor) o K (cinestésico). A fin de mantener el acrónimo original VARK, se conservaron las mismas iniciales para analizar y mostrar los resultados.

\section{Análisis estadístico}

Se realizó un análisis exploratorio de los datos para determinar conjuntos con características afines. Para ello se utilizó un análisis de conglomerados (cluster) jerárquico, usando las cuatro categorías VARK. Los datos del número total de veces que fue elegida cada opción se incluyeron en el programa estadístico SPSS v. 17.0. Tras obtener la solución cluster, se incluyó a cada encuestado en su correspondiente conglomerado y se calcularon las proporciones de cada estilo VARK en un gráfico comparativo.

Una característica del cuestionario VARK es que permite respuestas o elecciones múltiples en la misma pregunta, es decir, las opciones no son excluyentes. Esto trae aparejado que un encuestado pudiera elegir dos o más opciones que correspondieran, obviamente, a dos o más estilos diferentes, ya sea V, A, $\mathrm{R}$ o K. Por tanto, el resultado final suele contener un mosaico de opciones que requiere un análisis especial. Para ello se considera que un estilo es dominante cuando es elegido en más del 50\% de las respuestas. Cuando dos o más estilos alcanzan este porcentaje, la categoría corresponderá a un estilo bimodal (combinación de dos preferencias dominantes) o multimodal (combinación de tres o más preferencias dominantes). Para el caso de que ninguna opción $\mathrm{V}$, A, R o K alcance el 50\%, el umbral de aceptación se baja al 40\% (preferencia fuerte), lo que habitualmente resuelve el problema de inclusión en alguno de los estilos. De esta forma, cada encuestado tendrá un estilo preferente dominante o la combinación de algunos o todos ellos. El conteo y suma final de los resultados de cada individuo representará el estilo o estilos preponderantes de cada grupo (residentes, estudiantes de primer año o alumnos de séptimo grado). De esta forma se compararon los resultados entre los grupos y por sexo; en este último caso fue excluido el grupo de alumnos de educación primaria. Para el análisis se usó la prueba de hipótesis de chi al cuadrado para tablas $\mathrm{M} \times \mathrm{N}$ con el programa Epidat 2.1.

Este tipo de análisis, recomendado por los diseñadores del cuestionario VARK, se complementó con un análisis de correspondencia para determinar la afinidad de cada grupo estudiado por las dis-
Figura 1. Análisis de conglomerados (cluster) para determinar grupos de afinidad. En los cinco clusters encontrados se expresan las proporciones de los distintos estilos, junto al número de encuestados incluidos en cada conglomerado y su participación proporcional sobre el total. Para diferenciar los distintos grupos se tomó en cuenta la cantidad de veces que fue elegida cada opción, usando la muestra en conjunto de estudiantes de primer año y residentes $(n=254)$. La composición porcentual de los clusters se comparó con $\chi^{2}$ para tablas $5 \times 4(p<0,001)$. El conglomerado que agrupó a casi el $90 \%$ de los encuestados (cluster 1 ) estuvo compuesto por proporciones similares de V, A y R, y un poco más de K. Por su parte, el cluster 5 tuvo porcentajes parecidos al cluster 1 ; el resto de las combinaciones fueron infrecuentes (V: visual; A: auditivo; R: lectoescritor; K: cinestésico).

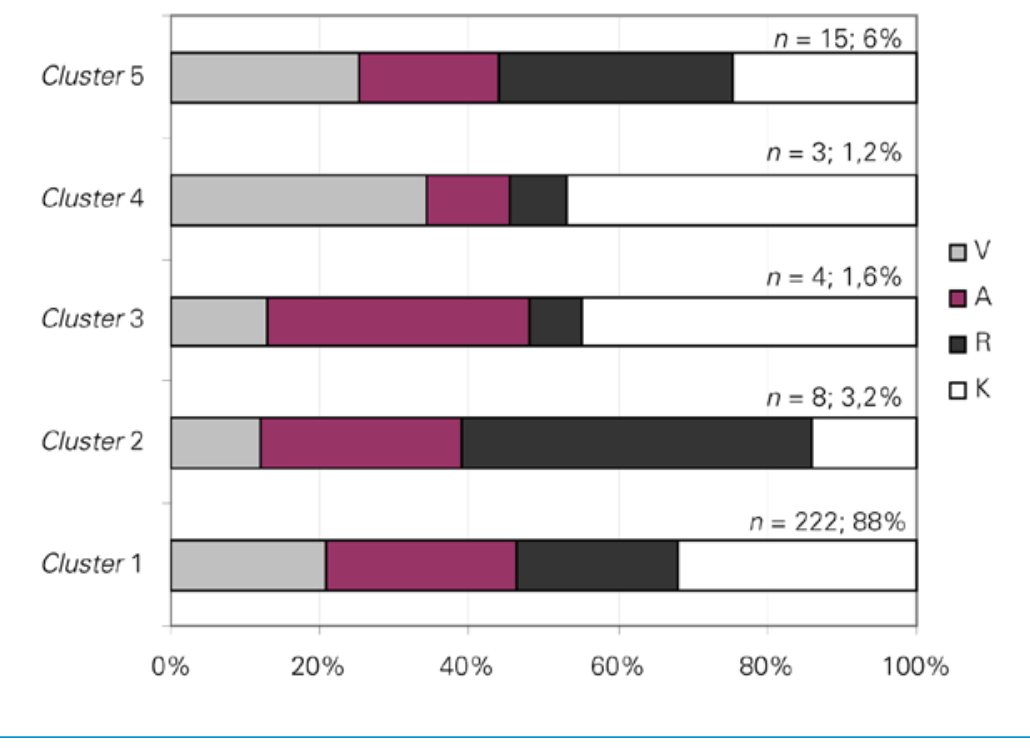

tintas categorías VARK. El mapa perceptual correspondiente para dos dimensiones se generó con el programa CorAna 2.1.

\section{Resultados}

Las medias de edad de cada grupo fueron las siguientes: residentes, 29,7 $\pm 2,97$ años; estudiantes de primer año, 20,6 $\pm 1,95$ años, y alumnos de primaria, 12,3 $\pm 0,5$ años.

El análisis cluster exploratorio sobre la muestra conjunta de estudiantes universitarios y de posgrado $(n=254)$ detectó cinco grupos con máximas diferencias entre sí (Fig. 1). El conglomerado más numeroso (cluster 1) estuvo compuesto por 222 encuestados (88\%) que presentaban afinidades similares para los cuatro tipos de estilos VARK. Un segundo conglomerado (cluster 5) contenía sólo 15 encuestados (6\%) con una conformación similar al cluster 1, excepto por una menor preferencia por el estilo A (auditivo) a expensas del crecimiento del perfil R (lectoescritor). Los otros tres conglomera- 
Figura 2. Distribución proporcional de los distintos estilos de aprendizaje obtenidos con el cuestionario VARK para cada uno de los grupos. Para la determinación de los porcentajes se consideró dominancia del estilo cuando éste era elegido en más del $50 \%$ de las veces; en caso de no alcanzar este nivel, se disminuyó el umbral al $40 \%$. La comparación estadística se realizó con $\chi^{2}$ para tablas $6 \times 3$ y $3 \times 2$; en este último caso sólo se muestran los valores de $p$ más bajos (V: visual; A: auditivo; R: lectoescritor; $\mathrm{K}$ : cinestésico).

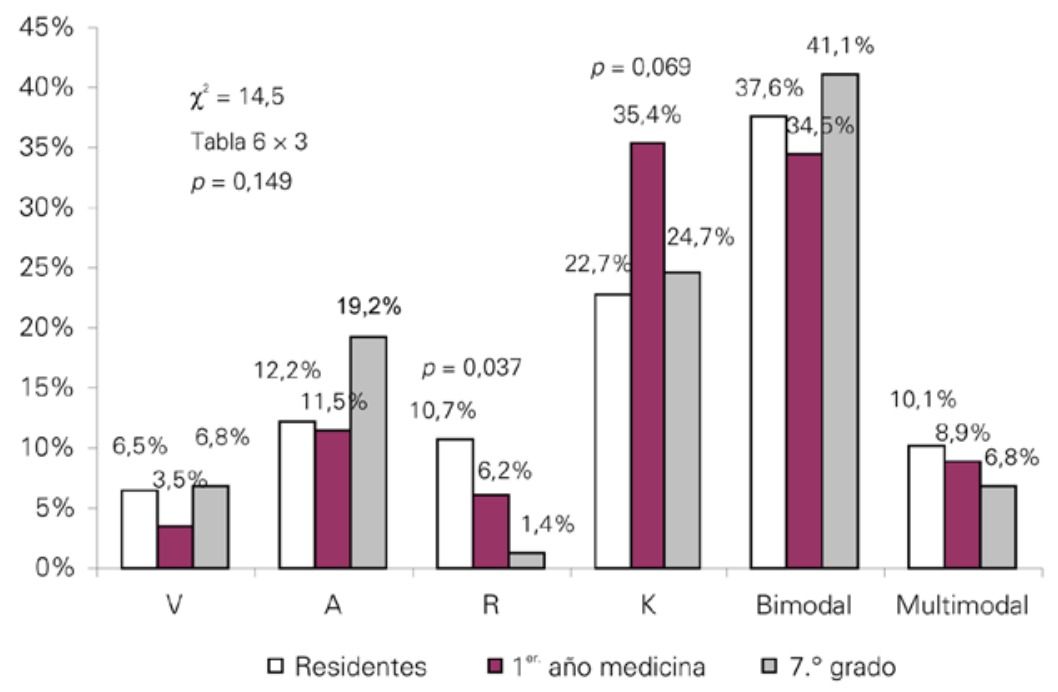

dos incluyeron en conjunto el 6\% restante de los encuestados, de acuerdo a distintas combinaciones o preferencias, como se observa en los clusters 2,3 y 4 de la figura 1.

La distribución proporcional de los distintos estilos de aprendizaje en cada grupo se muestra en la figura 2. En general, los perfiles preponderantes fueron el cinestésico (K), que rondó el $27,5 \%$, y el bimodal, con el 37,3\% (medias ponderadas). La comparación conjunta de los tres grupos (residentes, estudiantes de primer año y alumnos de primaria) no mostró diferencias estadísticas $(p=0,149)$; sin embargo, la comparación individual para cada perfil mostró un crecimiento significativo del estilo $\mathrm{R}$ (lectoescritor) desde la escuela primaria hasta el posgrado $(p=0,037)$. Las otras diferencias observadas en la figura 2 no mostraron peso estadístico, aunque ello podría depender de la escasa potencia de la muestra; en particular, podría existir una caída significativa de la preferencia auditiva (A) a partir del nivel primario hasta la universidad y el posgrado.

Al desagregar las combinaciones de estilos dentro de las modalidades bimodal y multimodal, se observó que la combinación preponderante era la AK (media ponderada: $43,4 \%$ ) y que esta diferencia disminuía significativamente desde la escuela primaria hasta el posgrado $(p=0,002)$ (Fig. 3).
Para la comparación por sexo de los estilos VARK se excluyeron los alumnos de la escuela primaria. La muestra de estudiantes estuvo compuesta por un $55,8 \%$ de mujeres, mientras que entre los graduados encuestados, las mujeres eran el 31,9\%. En la figura 4 se observa que, en términos generales, no hubo diferencia estadística entre los sexos, aunque se halló un mayor predominio del estilo $\mathrm{R}$ (lectoescritor) en los varones sobre las mujeres $(p=0,033)$ al realizar las comparaciones apareadas. Por su parte, las mujeres parecían tener mayor predilección por el estilo bimodal, a pesar de que con este tamaño de muestra no se alcanzó la significación estadística.

Por último, el análisis de correspondencia mostró la vinculación entre las distintas opciones VARK y los grupos estudiados. En el análisis bidimensional de vecindad entre las categorías y los grupos se observó que los alumnos primarios preferían lo auditivo (A), los estudiantes universitarios se acercaban más a lo cinestésico (K) y los encuestados del posgrado tenían una preferencia intermedia entre lo visual (V) y lo lectoescritor (R) (Fig. 5).

\section{Discusión}

En este estudio se individualizaron los estilos de aprendizaje de tres muestras de estudiantes desde la escuela primaria hasta el posgrado en medicina, en base a la elección de distintas modalidades perceptuales de adquirir y transmitir la información, a partir de las preferencias visual, auditiva, lectoescritora y cinestésica $[18,19]$. Los diferentes perfiles se obtuvieron con el cuestionario VARK, aplicado a 327 alumnos, a fin de conocer las preferencias en cada grupo de edad. Los estilos preponderantes fueron, en los tres grupos, el cinestésico (K) y el bimodal, con algunas diferencias en las preferencias por el perfil lectoescritor (R), que creció significativamente desde la escuela primaria hasta el posgrado, y probablemente una tendencia en la caída del estilo auditivo (A) desde aquel nivel educativo hasta la universidad y el posgrado $(p=0,269)$. Para el caso del estilo bimodal, la combinación preponderante fue la AK (auditivo-cinestésica), que disminuyó significativamente desde la escuela primaria hasta el posgrado.

El enfoque sobre los estilos de aprendizaje abordado en este estudio es únicamente una forma de analizar los perfiles o las preferencias de aprendizaje de los estudiantes. Los estilos descritos por Kolb [20], por ejemplo, incluyen dos formas opuestas de adquirir información -la conceptualización abstracta y la experiencia concreta- y dos maneras de usar- 
la -la observación reflexiva y la experimentación activa-. Otros trabajos utilizan la clasificación en estilos superficial, profundo y estratégico [12]. En particular, algunos estudios han encontrado que el estilo de aprendizaje superficial es el más común entre quienes ingresan en las escuelas canadienses de medicina [21]. Otros trabajos dividen los estilos en independiente, dependiente, colaborador, evitador, facilitador y competitivo [22,23]. Sin embargo, la herramienta VARK ha sido la más utilizada recientemente para determinar la forma de adquirir o entregar la información desde una modalidad sensorial [6,24-27]. En particular, los alumnos con preferencia por el estilo visual (V) acostumbran a usar diagramas, gráficos y símbolos. El estilo auditivo (A). en cambio, se inclina por escuchar explicaciones, asociar las ideas con anécdotas o historias, discutir los temas con maestros y compañeros, grabar las clases y leer en voz alta. Los estudiantes con perfil lectoescritor $(R)$ tienen preferencia por las listas, definiciones y glosarios, suelen usar más libros, revistas y consultas en Internet, pasan sus apuntes en limpio y reescriben las ideas con otras palabras. Por último, el estilo cinestésico prefiere los ejemplos concretos, ya sea con modelos, simulaciones o fotografías, las aplicaciones reales, el uso de algoritmos y problemas resueltos, y el ensayo de prueba y error.

Lujan y DiCarlo [6] estudiaron 155 alumnos de primer año de medicina con el cuestionario VARK. Encontraron que sólo el $36 \%$ de los encuestados prefería un modo único de presentación de la información, mientras que, en nuestro trabajo, la muestra de estudiantes de primer año presentó un perfil unimodal en el 56,6\%, sobre todo a expensas del crecimiento del perfil cinestésico (K). De igual forma, mientras que el estilo multimodal preponderante en el mismo trabajo fue el cuatrimodal (VARK), con un 43,4\%, el bimodal AK fue el más frecuente en nuestro grupo de estudiantes de pregrado. Baykan y Naçar [28], en Turquía, también aplicaron el VARK a 166 estudiantes de primer año de medicina. En este caso, la proporción de unimodalidad fue similar a la de Lujan y DiCarlo [6], pero al igual que en nuestro trabajo, dentro del perfil multimodal destacó el estilo bimodal.

Al comparar los sexos, Slater et al [24] y Nuzhat et al [25] encontraron que las estudiantes solían tener una mayor diversidad de combinaciones en sus preferencias de estilos con respecto a los varones. De la misma forma, Wehrwein et al [26] hallaron que la mayoría de los varones preferían una instrucción multimodal, especialmente cuatrimodal (VARK), y la mayoría de las mujeres, la instrucción unimodal,
Figura 3. Distribución en porcentajes y combinaciones de los distintos estilos de aprendizaje, de acuerdo al patrón multimodal, de residentes (a), estudiantes de primer año (b) y alumnos de séptimo grado (c). Las categorías corresponden a la combinación de visual (V), auditivo (A), lectoescritor $(\mathrm{R})$ y cinestésico $(\mathrm{K}) \cdot \chi^{2}=20,1$ para tablas $11 \times 3(p=$ $0,327)$; la comparación de las proporciones de AK entre los tres grupos mostró una $p=0,002$.
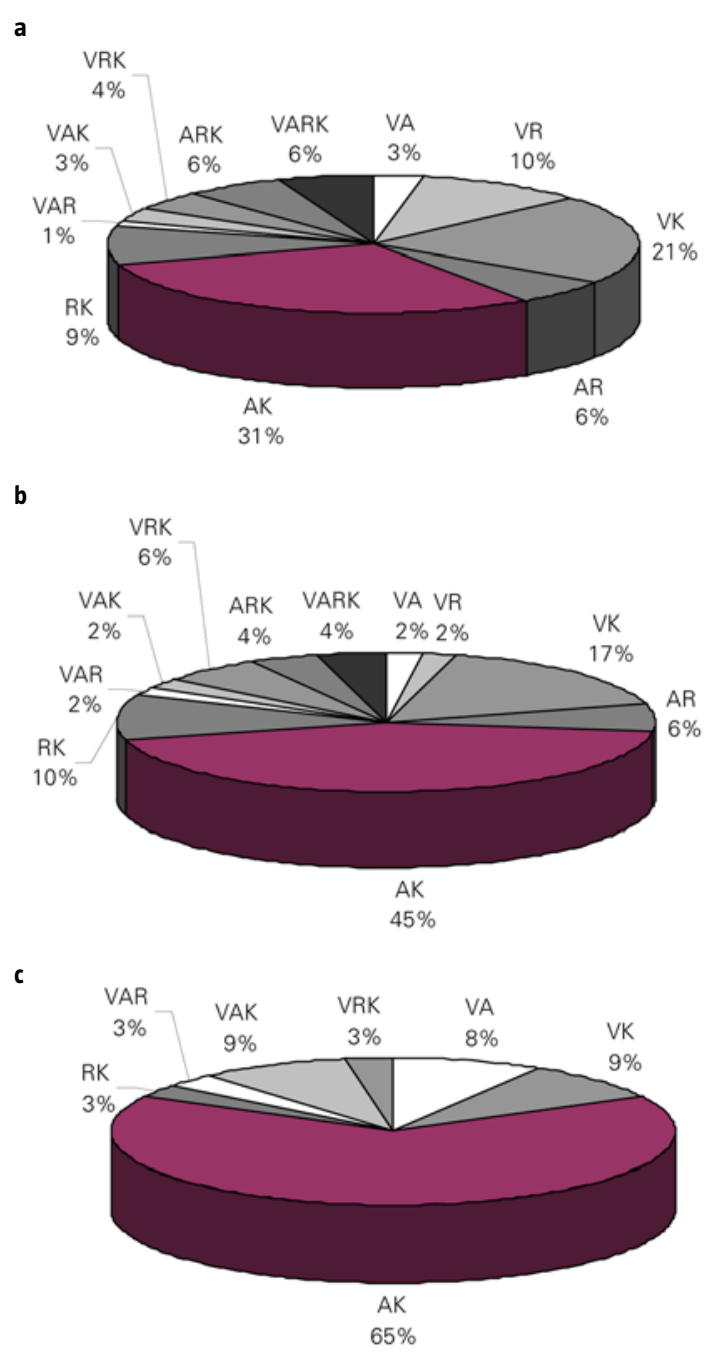

con predilección por el estilo cinestésico (K). A diferencia de estos autores, en nuestra investigación no se encontraron diferencias importantes entre los sexos, a excepción de una preponderancia del perfil lectoescritor ( $R$ ) en los varones. Como en nuestro caso, Baykan y Naçar [28] tampoco hallaron diferencias entre los sexos en estudiantes de medicina. Samarakoon et al [29] encontraron que un $69,9 \%$ de los estudiantes de primer año y un 67,5\% de los es- 
Figura 4. Distribución proporcional de los distintos estilos de aprendizaje obtenidos con el cuestionario VARK y divididos por sexo (excluidos los alumnos de séptimo grado). Para la determinación de los porcentajes se consideró dominancia del estilo cuando éste era elegido en más del 50\% de las veces; en caso de no alcanzar este nivel, se disminuyó el umbral al 40\%. La comparación estadística se realizó con $\chi^{2}$ para tablas $6 \times 2$ y $2 \times 2$ (V: visual; A: auditivo; R: lectoescritor; $\mathrm{K}$ : cinestésico).

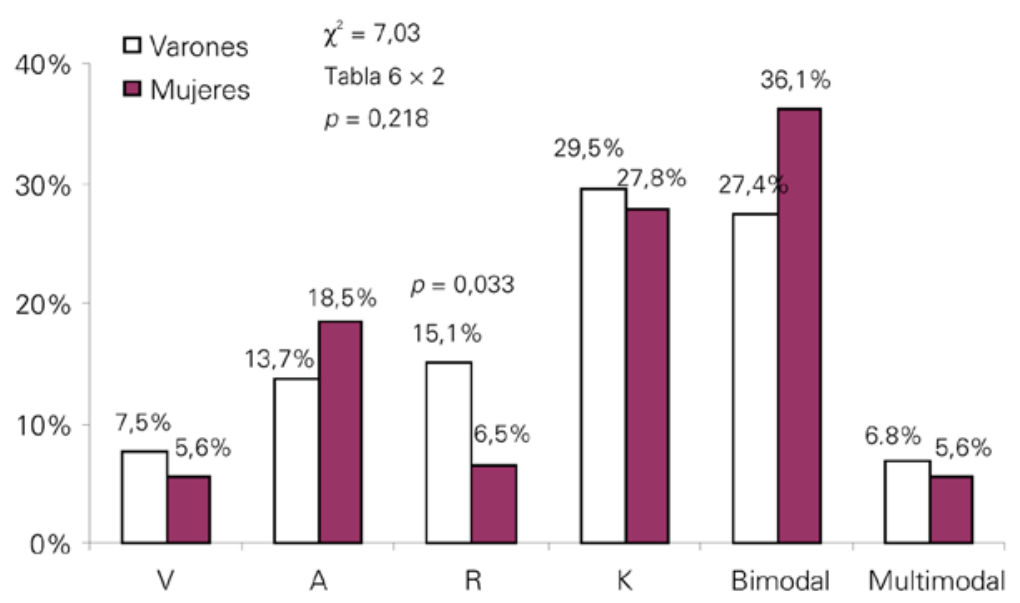

tudiantes del último año tenían estilos de aprendizaje multimodal, mientras que un $52,9 \%$ de los posgraduados tenían un perfil unimodal.

El análisis de correspondencia realizado en este estudio ofrece un enfoque complementario al análisis tradicional del cuestionario VARK. En el diagrama de correspondencia se muestra la afinidad entre las variables en base a la proximidad de las distintas preferencias de los grupos. A diferencia del análisis VARK basado en perfiles dominantes, para la determinación de las coordenadas de los estilos se tomó en cuenta sólo la cantidad de veces que fue elegida cada opción, sin tener en cuenta su dominancia. En el caso de los alumnos de primaria se observó una afinidad con lo auditivo (A), ya sea sólo o en combinación con otras modalidades, según se desprende del análisis previo. Los estudiantes encuestados se acercaron más al perfil cinestésico (K), sólo o en combinación, mientras que los residentes de cardiología se encontraron entre el estilo visual (V) y el lectoescritor (R), probablemente en combinaciones bimodales o multimodales.

Aunque las preferencias descritas con el cuestionario VARK no se relacionan con la motivación, la adaptación de la enseñanza al perfil preferido por los alumnos podría funcionar como una fuente de motivación para el aprendizaje. Conocer los estilos de aprendizaje en los distintos niveles de formación
Figura 5. Análisis de correspondencia para dos dimensiones. La proximidad de las distintas preferencias con los diferentes grupos indica la afinidad entre las dos variables. Para la determinación de las coordenadas de los estilos se tomó en cuenta la cantidad de veces que fue elegida cada opción.

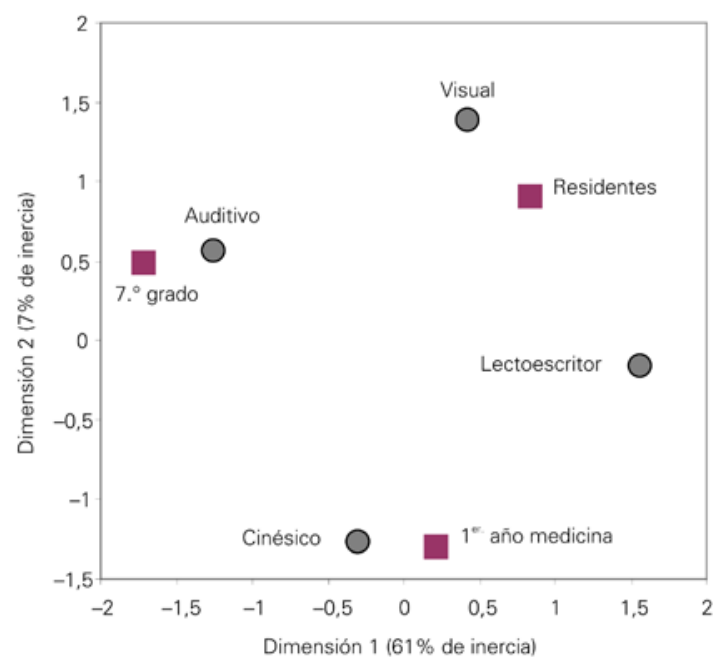

educativa puede ayudar a los propios alumnos a adoptar las mejores estrategias de estudio y ayudar a los docentes a elegir los abordajes de enseñanza más apropiados para motivar y facilitar la transmisión del conocimiento. Teniendo en cuenta que en este estudio se observó una fuerte preponderancia del estilo cinestésico (K) en los estudiantes universitarios, los mejores abordajes de la enseñanza en este nivel podrían incluir ejemplos concretos basados en modelos o en la realidad, simulaciones de aplicaciones reales, el uso de algoritmos y problemas resueltos, y ensayos de prueba y error. En futuros estudios longitudinales podría evaluarse el cambio en el desempeño académico de los alumnos tras la implementación de estas estrategias basadas en los estilos de aprendizaje. En el caso del grupo de posgrado llama la atención la preponderancia del perfil lectoescritor (R), mucho más común en los estudiantes y graduados de humanidades. Un estudio futuro podría evaluar los estilos preferidos por residentes de especialidades quirúrgicas, en donde sería posible encontrar una mayor preponderancia del perfil cinestésico (K).

Una limitación de este estudio es un probable sesgo de inclusión en la muestra de posgrado, ya que en este caso se eligieron sólo médicos que se hallaban en un programa de residencias clínicas (cardiología) en lugar de otra especialidad. Otra limitación 
es que, por tratarse de un estudio transversal, los grupos comparados fueron independientes. De forma ideal, un mismo grupo seguido a través de los años podría haber mostrado la verdadera evolución temporal de los estilos de aprendizaje. Por último, el método de identificación de los estilos de aprendizaje usado en este trabajo se basaba en un cuestionario, procedimiento comúnmente conocido como 'autoinforme' (self-reports). Algunos estudios en otras disciplinas han sugerido que los autoinformes podrían ser menos fiables que otro tipo de herramientas basadas en la observación directa del educando [9], lo que debería tenerse en cuenta como otro límite potencial de este estudio.

En conclusión, en este trabajo se individualizaron los estilos de aprendizaje de tres muestras de estudiantes desde la escuela primaria hasta el posgrado en medicina, de acuerdo a las distintas modalidades sensoriales descritas en el cuestionario VARK. Los estilos preponderantes en los tres grupos (escuela primaria, universidad y posgrado) fueron el cinestésico $(\mathrm{K})$ y el bimodal, con algunas diferencias en las preferencias por el perfil lectoescritor (R), que creció significativamente desde la escuela primaria hasta el posgrado, y probablemente una tendencia en la caída del estilo auditivo (A) desde el mismo nivel de primaria hasta el posgrado. Por su parte, la combinación bimodal preponderante fue la AK (auditivo-cinestésica), que disminuyó significativamente desde la escuela primaria hasta el posgrado. Por último, no se encontraron diferencias importantes entre los sexos tanto en el nivel universitario como en el posgrado inmediato. Esta información podría servir para adaptar las condiciones y métodos de enseñanza al perfil preferencial de los estudiantes.

\section{Bibliografía}

1. Tanner K, Allen D. Approaches to biology teaching and learning: learning styles and the problem of instructional selection-engaging all students in science courses. Cell Biol Educ 2004; 3: 197-201.

2. James W, Gardner D. Learning styles: implications for distance learning. New Dir Adult Contin Educ 1995; 67: 19-32.

3. Bitran $M$, Lafuente $M$, Zúñiga $D$, Viviani $P$, Mena $B$. The influence of psychological features and learning styles on the academic performance of medical students. Rev Med Chil 2004; 132: 1127-36.

4. Bitran M, Zúñiga D, Lafuente M, Viviani P, Mena B. Psychological types and learning styles of students entering medical school at the Pontificia Universidad Católica de Chile. Rev Med Chil 2003; 131: 1067-78.
5. Canaleja-Pérez MC, Martínez-Martín ML, Pineda-Ginés MC, Vera-Cortés ML, Soto-González M, Martín-Marino A, et al. Estilos de aprendizaje en los estudiantes de enfermería. Educ Med 2005; 8: 83-90

6. Lujan HL, DiCarlo SE. First-year medical students prefer multiple learning styles. Adv Physiol Educ 2006; 30: 13-6.

7. Cook DA. Reliability and validity of scores from the index of learning styles. Acad Med 2005; 80: S97-101.

8. Armstrong E, Parsa-Parsi R. How can physicians' learning styles drive educational planning? Acad Med 2005; 80: 680-4.

9. Veenman MV, Prins FJ, Verheij J. Learning styles: self-reports versus thinking-aloud measures. Br J Educ Psychol 2003; 73: 357-72.

10. Rohrer D, Pashler H. Learning styles: where's the evidence? Med Educ 2012; 46: 634-5.

11. Kalaca S, Gulpinar M. A Turkish study of medical student learning styles. Educ Health (Abingdon) 2011; 24: 459.

12. McManus IC, Richards P, Winder BC, Sproston KA. Clinical experience, performance in final examinations, and learning style in medical students: prospective study. BMJ 1998; 316: 345-50.

13. Fleming ND. VARK, a guide to learning styles. URL: http://www. vark-learn.com/english/page.asp?p=questionnaire. [01.10.2009].

14. Linares AZ. Learning styles of students and faculty in selected health care professions. J Nurs Educ 1999; 38: 407-14.

15. Ramsden P. Student learning and perceptions of the academic environment. High Educ 1979; 8: 411-28.

16. Gurpinar E, Bati H, Tetik C. Learning styles of medical students change in relation to time. Adv Physiol Educ 2011; 35: 307-11.

17. Engels PT, De Gara C. Learning styles of medical students, general surgery residents, and general surgeons: implications for surgical education. BMC Med Educ 2010; 10: 51.

18. Morse JS, Oberer J, Dobbins JA, Mitchell D. Understanding learning styles: implications for staff development educators. J Nurs Staff Dev 1998; 14: 41-6.

19. Curry L. Cognitive and learning styles in medical education. Acad Med 1999; 74: 409-13.

20. Kolb D. Experiential learning: experience as the source of learning and development. New Jersey: Prentice Hall; 1984.

21. Aaron S, Skakun E. Correlation of students' characteristics with their learning styles as they begin medical school. Acad Med 1999; 74: 260-2.

22. Grasha A. Teaching with style. A practical guide to enhancing learning by understanding and teaching styles. New York: Alliance Publishers; 1996.

23. Vaughn L, Baker R. Teaching in the medical setting: balancing teaching styles, learning styles and teaching methods. Med Teach 2001; 23: 610-2.

24. Slater JA, Lujan HL, DiCarlo SE. Does gender influence learning style preferences of first-year medical students? Adv Physiol Educ 2007; 31: 336-42.

25. Nuzhat A, Salem RO, Hamdan NA, Ashour N. Gender differences in learning styles and academic performance of medical students in Saudi Arabia. Med Teach 2013; 35 (Suppl 1): S78-82.

26. Wehrwein EA, Lujan HL, DiCarlo SE. Gender differences in learning style preferences among undergraduate physiology students. Adv Physiol Educ 2007; 31: 153-7.

27. Alkhasawneh E. Using VARK to assess changes in learning preferences of nursing students at a public university in Jordan: implications for teaching. Nurse Educ Today 2013; 33: 1546-9.

28. Baykan Z, Naçar C. Learning styles of first-year medical students attending Erciyes University in Kayseri, Turkey. Adv Physiol Educ 2007; 31: 158-60.

29. Samarakoon L, Fernando T, Rodrigo C. Learning styles and approaches to learning among medical undergraduates and postgraduates. BMC Med Educ 2013; 13: 42. 